\title{
Dimensioning the Future Pan- European Optical Network with Energy Efficiency Considerations
}

\author{
Anna Tzanakaki, Kostas Katrinis, Tanya Politi, Alexandros Stavdas, Mario Pickavet, Peter Van Daele, \\ Dimitra Simeonidou, Mike J. O’Mahony, Slavisa Aleksic, Lena Wosinska, Paolo Monti
}

\begin{abstract}
This paper studies the overall energy consumption of a Pan-European optical transport network for three different time periods: today, in five and ten years from now. In each time period the PanEuropean network was dimensioned, using traffic predictions based on realistic data generated by the optical networking roadmap developed in the framework of the European project Building the Future Optical Network in Europe - BONE. A wavelength routed WDM optical network based on either transparent or opaque node architectures was examined considering exclusively either 10Gbit/s or 40Gbit/s per channel data rates. The results manifest that transparent optical networking technologies are expected to provide significant energy savings of the order of $35 \%$ to $55 \%$. It was also shown that the migration towards higher data rates, i.e. from $10 \mathrm{Gbit} / \mathrm{s}$ to $40 \mathrm{Gbit} / \mathrm{s}$, is assisting to improve the overall energy efficiency of the network.
\end{abstract}

Index Terms-Optical fiber networks; WDM networks; energy efficiency

Manuscript received December 6, 2010

A. Tzanakaki is with the Athens Information Technology, Peania, 19002 Greece (phone: 303-555-5555; fax: +302106682766; email: atza@ait.edu.gr).

Kostas Katrinis was with Athens Information Technology, Greece. He is now with Dublin Research Lab, IBM Research, Dublin, Ireland (e-mail: katrinisk@ie.ibm.com).

T. Politi is with the University of Peloponnese, Greece (e-mail: tpoliti@uop.gr )

A. Stavdas is with the University of Peloponnese, Greece (e-mail astavdas@uop.gr )

M. Pickavet is with the Ghent University - IBBT - IMEC, Belgium (e-mail: mario.pickavet@intec.ugent.be)

P. Van Daele is with the Ghent University - IBBT - IMEC, Belgium (e-mail: peter.vandaele@intec.ugent.be)

D. Simeonidou is with the University of Essex, UK (e-mail: dsimeo@essex.ac.uk)

M. J. O'Mahony is with the University of Essex, UK (e-mail: mikej@essex.ac.uk)

S. Aleksic is with the Vienna University of Technology, Austria (e-mail: slavisa.aleksic@tuwien.ac.at)

L. Wosinska is with the KTH - Royal Institute of Technology, Sweden (email: wosinska@kth.se)

P. Monti is with the KTH - Royal Institute of Technology, Sweden, (e-mail: pmonti@kth.se)

\section{INTRODUCTION}

A $\mathrm{S}$ the Internet and ICT usage is becoming more and more omnipresent in today's society, its power consumption becomes also of increasing significance. As indicated in [1], ICT (comprising networks and network terminal equipment such as PCs, servers, TVs, etc.) is responsible for about $4 \%$ of all primary energy today worldwide, and this percentage is expected to double by 2020. Network equipment plays a significant role in this consumption figures. While access networks are responsible for a major part of the network power consumption today, recent studies (e.g. [2]) predict that the power share of the core network segment will grow rapidly. This will cause severe problems, not only in terms of resulting greenhouse gas emissions, but also because of the more complex and expensive cooling systems needed to cool router and switching equipment at ever higher heat production per square inch. These severe problems call for special research attention towards more energy-cautious core network solutions.

It is clear that broadband access penetration increases rapidly, and higher bandwidth access technology such as fiber to the X (FTTX) or even fiber to the home (FTTH) is becoming available to the end users. In addition, increased data and IP traffic, specifically traffic generated by emerging applications such as e-science, e-business, elearning, e-health and e-government, business services (such as IP, VPN, VoIP and IP videoconference) and residential services (such as triple play, IPTV and online gaming) are shaping up the requirements that the network of the future needs to support. It is also virtually certain that this traffic growth will continue both in the near future and in the longer term, due to emerging and new services offered to the end users, with demanding requirements in terms of network accessibility, capacity and functionality. This traffic growth that is initially observed and associated with the access part of the network is effectively propagating and affecting the transport network segment. This is mainly due to the fact that a portion of this volume of traffic needs to be exchanged between geographically remote locations interconnected through the transport 
network. This increased capacity requirement will have a direct impact on the energy consumption of the corresponding network segment and will be very dependent on the architecture and technology used to support it. It is clear that optical networking has been identified as the solution of choice, to support the transport segment of the network of the future. This is due to the bandwidth abundance it provides through technologies such as wavelength division multiplexing (WDM), the long transmission distances it supports, the improved flexibility, transparency and cost efficiency it offers. An additional benefit this technology option is offering compared to its conventional optoelectronic counterparts is improved energy efficiency. It is evident that the level of energy efficiency that can be achieved through optical networking is also very dependent on specific architectural approaches followed [3], [4], [5], technology choices made [6] as well as use of suitable algorithms and provisioning schemes [7]. Recognizing the importance of these parameters several approaches have been proposed in the literature aiming at reducing the energy consumption of network infrastructures [3] - [7].

To the best of the authors' knowledge, this paper is aiming for the first time at evaluating the energy savings achievable through the use of wavelength routed optical transport networks in a realistic framework of the network of the future. More specifically, the energy consumption results presented in this paper are obtained calculating the energy consumption of the optical networking equipment required in a Pan-European telecommunications network. These equipment requirements are identified through a network dimensioning study carried out considering current needs, with regards to bandwidth requirements, as well as future needs in the near and longer term i.e. the next five and ten years respectively. This study is based on the COST 239 [8] reference network topology. It takes, as input traffic matrix, a set of traffic demands that are generated as part of the development of the European Optical Networking Roadmap. This input traffic data has been used in order to offer a realistic approach in modeling the network of the future at a European scale. This roadmap is produced in the framework of the European Network of Excellence "Building the Future Optical Network in Europe" - BONE funded by the European Union [9]. The BONE consortium, recognizing that optical networking is the wired technology of choice to support the network of the future, is focusing on various aspects of optical networking. In order to study in more detail the expected growth of telecommunications networks and to identify suitable technology choices, BONE has focused part of its wide activities in sketching a PanEuropean "roadmap" for optical networking. The term "roadmap" here describes a generic direction for technology development or usage. The roadmapping process provides a way to identify, evaluate, and select strategic alternatives that can be used to achieve a desired technology or business objective. In this context, the roadmap work in BONE aims at producing realistic predictions, with regards to capacity requirements, in the network of the future. These can be used to identify optimum architectural approaches and technology solutions considering performance and other service and network level constraints as appropriate.

In the scope of this paper, the roadmap output regarding the scale and type of the future Pan-European network will be examined from the point of view of energy consumption. A relevant comparison between traditional optoelectronic solutions and all-optical approaches will be carried out. Our results indicate that transparent optical networks provide a clear benefit with regards to energy efficiency compared to their optoelectronic counterparts. Considering the expected traffic growth over the next decade, the relevant impact further increases providing energy savings that vary between $35 \%$ and $55 \%$ of the overall Pan-European transport network power consumption over time. This energy saving variation is dependent on the traffic volume supported by the network during the different time periods considered, the different technology options examined as well as the per channel data rates (network switching granularity) used.

The rest of the paper is structured as follows: section II discusses the roadmapping methodology used with the aim to produce a realistic traffic matrix for the current and the future Pan-European network. Section III presents the node architectures considered and the specific power consumption models used. Section IV discusses the details of the network dimensioning study, while section $\mathrm{V}$ provides the power dissipation results and discusses the relevant trends. Finally section VI summarizes the conclusions of the work presented in this paper.

\section{TRAFFIC PROJECTION METHODOLOGY AND RESUlts}

Trying to follow a realistic approach in predicting the capacity required by the European Network of the Future the BONE optical networking roadmap takes as input the access bandwidth as well as the services and applications available to the end users currently and their expected evolution in the next five and ten years on a per European country basis. The methodology followed in the roadmap development is illustrated in Figure 1. In more detail, information regarding existing and emerging applications and services available to end users, broadband penetration, existing and upcoming access technologies was collected for various European countries through a detailed survey performed among various BONE partners [10], [11]. This information was then processed taking into consideration the relevant country level demographics. The digital European diversity was considered in the future prediction of service and technology deployment as well as the relative population percentage, as densely populated countries is more likely to take on new technologies in an accelerated way. Densely populated areas even in countries that lack specific technology deployment will be more likely to deploy new services. A detailed description of specific case studies examined can be found in [11].

Based on the per-country input data, and taking into consideration the parameters described above, an analysis, 
the details of which can be found in [11], was performed to produce the traffic generated/terminated and supported by the various European countries under consideration. A percentage of this traffic is effectively fed and has to be supported by the Pan-European network. It was assumed that $20 \%$ of the traffic that is generated within each country is fed and will be serviced by the Pan-European network. Based on this assumption a matrix indicating the volume of traffic that will enter the Pan-European network and will be associated with each European country considered in the analysis was produced. Each European country considered is represented by the corresponding capital as a source/destination node thus forming the topology of the optical transport network under study. The source/destination information, in combination with the traffic data associated with it, is then converted into a conventional traffic matrix to be used for the Pan-European network dimensioning that will follow. This traffic matrix was obtained from the in-/out-bound traffic projections per source/destination site, assuming wavelength granularity of demands and uniform distribution of traffic to and from each node to all other nodes. The study focused on a subset of all potential European source/destination nodes of the core traffic, according to the Pan-European reference network topology proposed by the COST 239 action.

\section{POWER CONSUMPTION ESTIMATION FRAMEWORK}

As already discussed the estimation of energy consumption of a telecommunications network is highly sensitive to the network architecture employed and the technology used. Considering the continuing migration towards service-centric networks, it is expected that the European optical transport network will migrate towards a high-speed, highly reconfigurable WDM architecture as described above. A very promising candidate in this context would be a wavelength routed WDM network supporting dynamic network re-configurability with wavelength switching granularity through the use of Optical CrossConnects (OXC's). Considering this scenario, it is clear that the overall network power consumption will be determined by the architecture and technology choices of the optical cross-connect nodes as well as the configuration and technology solutions of the fiber links.

In this study, a WDM optical network architecture based on Wavelength Selective Switching (WSS) nodes without wavelength conversion [12] is assumed, as it offers a scalable approach that can support the very high capacity requirements predicted for the near and more so the longer term future of the Pan-European network [11]. The specific node architecture considered is shown in Figure 2, employing wavelength-selective switches using MicroElectrical Mechanical Systems (MEMS). The input/output fibers of the OXC's are supporting a maximum of $\mathrm{W}$ wavelengths each, which is set to 40 in this study. To provide comparative results and conclusions regarding the energy consumption of transparent vs. optoelectronic solutions, two alternative OXC technology options are evaluated: one supporting a transparent optical network approach, where data remains in the optical domain while traversing the optical path without optoelectronic conversions at any intermediate node (Figure 2a), and one that supports the opaque network architecture. In the latter case the optical signal is converted into the electronic domain at every intermediate node along each lightpath (Figure $2 \mathrm{~b}$ ). It should be noted that in the opaque OXC case the optical switching fabrics are surrounded by optoelectronic interfaces converting the signal from optical to electrical and back to optical in order to offer regeneration of the signal, reducing the effect of transmission and switching impairments present in alloptical networks. These interfaces are formed by receiver and transmitter pairs that can be classified into two groups: a) receivers and transmitters that are directly interfacing the transmission line and therefore are commonly known as long reach transceivers and b) receivers and transmitters that are interfacing the optical switch fabric known as short reach transceivers. Regarding the fiber links a model comprising a sequence of alternating Single Mode Fiber (SMF) and Dispersion Compensation Fiber (DCF) spans, is assumed to address fiber dispersion effects. To compensate for the insertion loss of the fiber spans optical amplifiers based on Erbium Doped Fiber Amplifier (EDFA) technology are allocated at the end of each transmission span. In Figure 2, all power-consuming (active) elements are shown in grey color, namely the MEMS switches, the optical amplifiers allocated at each incoming/outgoing fiber to compensate for fiber and other optical component insertion loss and the transmitter/receiver pairs. It should be noted that the two classes of receiver/transmitter pairs located at the opaque OXC nodes have different energy consumption levels. More specifically the long reach compared to short reach receiver/transmitter pairs require higher power dissipation. Table I lists the power consumption values (in Watts) that have been assumed for the power-dissipating (active) devices to be used for the calculation of the overall network power consumption. These are typical consumption figures originating mostly from datasheet surveys. The power consumption of larger 3D MEMS $(\mathrm{N}>64)$ has been approximated as a linear function of the MEMS size.

Additionally to power consumption due to active devices, we also incorporate power dissipation due to cooling in the power estimation mode used and a $100 \%$ power overhead due to cooling [13] is assumed. It should be noted that all energy consumption calculations account only for the transport equipment of the European network, the power dissipated by electronic circuits, such as control boards of $\mathrm{OXCs}$ or hardware implementing protocol functionality is not considered.

\section{NETWORK DIMENSIONING FRAMEWORK}

As already discussed the energy consumption of the PanEuropean optical network is based on the calculation of the energy dissipation of the network equipment involved. The equipment requirements of this network are calculated through a network dimensioning study that takes as input the predicted traffic demands produced by the BONE 
roadmap.

The calculation of the dimensions of the European network, for the three periods under consideration, is performed through solving an instance of the network dimensioning problem referred to as "Brownfield Network Dimensioning". In this version of the problem, the geographical location of nodes is given together with the set of trenched physical links (ducts) connecting neighboring nodes. The output of the dimensioning process is the optimal number of fibers per link and wavelengths per fiber that need to be installed to serve the input traffic matrix at minimum cost, as well as the optimal dimensions of optical switches required to transparently route the input traffic.

The adopted method employs a linear cost model incorporating fiber cost (fixed and length-dependent components), Wavelength cost and size-dependent MEMS switch cost. In addition, all flow constraints are linear, thus admitting the formulation of the problem as a linear programming problem with integer solutions, similarly to the approach reported in [14]. Each source-destination demand (expressed at wavelength granularity) is served by $\mathrm{k}=3$ candidate lightpaths using standard k-shortest path routing, whereby physical distance is used for assigning link weights during the routing process. Since the goal of the dimensioning study is to capture future trends rather than specify exact quantitative requirements, we considered only a subset of all potential European source/sinks of core traffic. Specifically, we adopted the Pan-European test network topology proposed by the COST 239 action, comprising 10 nodes ("Zurich" node excluded due to unavailability of traffic data for this site) and 42 unidirectional physical links.

The results produced both from a network dimensioning and energy consumption perspective are of particular interest, as they are based on a realistic evaluation of the current and the future traffic demands that the PanEuropean transport network has to support, generated through the BONE optical networking roadmap activity (cf. section II). Specifically, using the obtained aggregate in/outbound traffic projection per site (expressed in Tbit/s), we generated traffic matrices, assuming wavelength granularity of demands, 40 wavelengths per fiber and uniform distribution of traffic to/from a node to all other nodes. For three input datasets of estimated aggregate traffic per site, corresponding to three distinct time intervals, i.e. currently, in five years and in ten years from today, we generated traffic matrices assuming in one case $10 \mathrm{Gbit} / \mathrm{s}$ and in a second case $40 \mathrm{Gbit} / \mathrm{s}$ data rates per wavelength channel. Through this process, six input traffic matrices were obtained in total. Thus, forming 12 discrete network scenarios to be examined and compared with regards to the total power consumption. These scenarios are the following: current, near future (five years from today) and long term future (ten years from today) network supporting 10Gbit/s per wavelength channel (switching granularity) for both cases the transparent and opaque OXC architectures and also current, near future (five years from today) and long term future (ten years from today) network supporting 40Gbit/s per wavelength channel (switching granularity) for both cases the transparent and opaque OXC architectures.

Using the dimensioning method and the input parameter set outlined above, we created instances of Integer Linear Programs and solved each instance to optimality using the Gurobi solver [15]. This enabled us to specify the minimumcost dimensions of the European optical core network corresponding to the present, as well as to its projected future evolution in the near and the longer term.

Figure 3 depicts two key metrics that are relevant to the evolution of the dimensions of the European backbone, i.e. aggregate number of lightpaths and average number of fibers installed per physical link for both the 10Gbit/s and the $40 \mathrm{Gbit} / \mathrm{s}$ solutions. The migration from 10Gbit/s to $40 \mathrm{Gbit} / \mathrm{s}$ data rates noticeably limits the dimensions of the optical network in terms of number of fibers per link (reducing the requirements for the associated equipment i.e. optical amplifiers and multiplexing/de-multiplexing equipment) and as such reduces the incurred costs. In the 10Gbit/s case a significantly larger number of fibers per link are required particularly in the five years from today and beyond time frame. This is due to that in the 40Gbit/s case the total number of lightpaths that need to be established is significantly reduced compared to the 10Gbit/s case. These results indicate that in order to downscale the dimension of the future Pan-European network high data rate solutions should be used. However, it is important to note that there is a trade-off between the benefit in the network dimension and the per-channel data rate that the network supports. This trade-off is associated with two parameters: one being the efficiency with which the network resources are utilized, when high data rates are supported, and the transparency distance that can be attained in these networks. By transparency distance we refer to the longest transmission distance feasible before regeneration of the optical signal is required. In more detail, when the per-channel data rate increases, depending on the traffic distribution, wavelength channels may start being underutilized, thus effectively limiting the level to which the network can be downscaled. This effect is also dependent on the granularity of demands and will be accompanied by a cost and an energy consumption penalty, associated with the higher rate equipment required in this case. In addition, at higher data rates transmission and switching impairments have a greater impact on the signal quality. Therefore, higher data rates, in general, further limit the transparency distance that a signal can traverse as well as the number of channels that can be supported within one fiber. In the literature it has been reported that these effects can be addressed, to some extent, using advanced technologies involving alternative modulation formats [16], error correction techniques [17], etc. Therefore, although there have been some demonstrations of practical systems for long-reach 100 Gbit/s per-channel transmission recently, these systems may not be currently mature enough or commercialized to a sufficient extent. In this context, they are not yet optimized for power consumption and including them in a comparative study may lead to inaccurate conclusions. Taking into account these points, this work did not consider higher perchannel data rates, such as 100Gbit/s and beyond, although they may present a practical approach for the long term 
future [18].

\section{Power Estimation Results And Discussion}

Based on the dimensions of the European optical network derived as described above the calculation of its present and future energy footprint is performed as a post-processing step following the power consumption estimation framework outlined in Section III. The results produced clearly indicate that the transparent approach offers significant energy savings compared to its optoelectronic counterpart. An evaluation of the benefits and limitations of different data rates supported on a wavelength basis with regards to energy efficiency was also carried out. More specifically Figure 4, illustrates the total network power consumption for the two OXC architectures under study, i.e. for the transparent and opaque case, supporting two possible per channel data rates (determining the corresponding switching granularities) namely $10 \mathrm{Gbit} / \mathrm{s}$ and $40 \mathrm{Gbit} / \mathrm{s}$. Power consumption results are produced for all three time periods under consideration i.e. currently, after five and ten years from today. Figure 4, also indicates the volume of aggregate traffic that is supported by the Pan-European network during each time period of study.

It can be clearly seen that the transparent approach is offering an energy saving that remains above $35 \%$ compared to the opaque approach for all three periods and for the 10Gbit/s per channel data rate case. The energy saving percentage obtained through the transparent approach is somewhat increased for the $40 \mathrm{Gbit} / \mathrm{s}$ per channel data rate for which it remains above $55 \%$ for all three periods examined. Considering the growth of the absolute figures of network power consumption with time this is a very significant saving. In both data rates examined, the energy savings observed are associated with the reduced number of transceiver requirements obtained in the transparent case compared to that corresponding to the opaque case.

Figure 4 also shows that the 40Gbit/s per channel data rate approach for the transparent OXC architecture significant energy savings are gained compared to the corresponding 10Gbit/s transparent solution. However, it should be noted that this is achieved at the expense of a lower network switching granularity that may affect the efficiency of network resource utilization in the common case where sub-wavelength traffic demand granularity applies.

In order to provide some more insight on the driving factors that determine the overall network power consumption for the transparent node architecture considering both $10 \mathrm{Gbit} / \mathrm{s}$ and $40 \mathrm{Gbit} / \mathrm{s}$ per wavelength channel data rates, some additional results were produced (Figure 5). The results were taken for the near (five years form today) and the longer term future (ten years from now) time periods. As can be seen in Figure 5, for the near term case and the $10 \mathrm{Gbit} / \mathrm{s}$ per channel data rate the main power dissipating elements are the transceivers, with the required EDFAs following quite closely in terms of their overall power consumption. The least energy consuming elements appear to be the optical switches based on MEMS technology. For the 40Gbit/s per channel data rate and for the same period the general trend is quite similar. Therefore, also in this case, the power consumption is higher for the transceivers, somewhat lower for the EDFAs and even lower for the optical switches. As can be seen the transceivers, the EDFAs and the switches overall power dissipation in this second case are significantly lower than that of the 10Gbit/s case, which is in line with the results shown in Figure 4. In more detail, as expected, in the higher per channel data rate case, traffic demands are accommodated by a reduced number of lightpaths (Figure 3) leading to a decrease in network equipment requirements. As in this analysis we have assumed a maximum of 40 wavelengths supported by each fiber the reduced dimension is mainly reflected on reduced number of fibers per link. This is then directly translated into not only reduced transceiver count, but also number of optical amplifiers and number of active optical switch ports. The influence on the switch port count is associated with the OXC node architecture used, as in the WSS architecture considered the individual MEMS switch dimensions are determined by the number of fibers supported by the node. It is interesting to note that when migrating from 10Gbit/s to $40 \mathrm{Gbit} / \mathrm{s}$ per channel rate the power consumption of transceivers becomes even lower than the amplifier power consumption observed in the low per channel rate. Figure 5 also shows the details of power consumption for the various active network elements in case of $10 \mathrm{Gbit} / \mathrm{s}$ and $40 \mathrm{Gbit} / \mathrm{s}$ data rates for the longer term future. The overall trend in this case is very similar to the one observed for the near term analysis, but magnified in terms of absolute power consumption figures.

\section{CONCLUSION}

The work presented in this paper is attempting to quantify the overall network power consumption of the future European network. In order to provide a realistic basis for this analysis the Pan-European telecommunications network was examined as a test case for three different time periods: today, in the next five and ten years. Dimensioning of the Pan-European network was performed for the three time periods considered, assuming a wavelength routed WDM optical network. Two different architectural choices were examined one employing transparent OXC nodes utilizing the wavelength selective approach and the second utilizing the wavelength selective architecture, but adhering to an opaque solution including optoelectronic conversions at the input and output of the switching fabrics incorporated at each node. This dimensioning was based on traffic matrices produced through current broadband access penetration, technology and associated service information as well as relevant future predictions that were collected for different European countries. These were processed according to the optical networking roadmap methodology developed in the framework of the European project BONE. Based on the output of this dimensioning exercise and using specific 
energy consumption models for the node and link architectures assumed the overall energy consumption of the Pan-European network was calculated for the two architectures under consideration. The network topology used was based on the COST 239 reference network. The results have shown that the overall power consumption of the Pan-European network is quite significant and considering the expected network growth required to accommodate future service requirements it is expected that it will keep increasing very fast for the next decade, almost quadrupling its value. The use of transparent optical networking technologies is expected to provide significant energy saving in the network and our results have shown that this can vary between $35 \%$ to $55 \%$ of the overall power consumption requirements of the network. The use of higher per channel data rates in the transparent approach is shown to have an impact in the overall energy efficiency of the network due to the reduced equipment requirements it introduces.

\section{ACKNOWLEDGMENT}

This work was performed under the support of the BONE-project ("Building the Future Optical Network in Europe"), a Network of Excellence funded by the European Commission in the 7th ICT- Framework Program.

\section{REFERENCES}

11 M. Pickavet, W. Vereecken, S. Demeyer, P. Audenaert, C. Develder, D. Colle, B. Dhoert and P. Demeester, "Worldwide energy needs of ICT: the rise of power-aware networking”, proceedings IEEE ANTS 2008, Mumbai (India), pp. 15-17, Dec. 2008

[2] J. Baliga, K. Hinton, R. S. Tucker, "Energy consumption of the Internet", proceedings COIN-ACOFT 2007, Melbourne (Australia), pp 1-3, June 2007

[3] G. Shen and R. S. Tucker, "Energy-Minimized Design for IP Over WDM Networks”, Journal of Optical Communications and Networking, vol 1, no. 1, pp. 176-186, June 2009.

[4] A. Stavdas, C. (T) Politi, T. Orphanoudakis, and A. Drakos, "Optical packet routers: how they can efficiently and cost-effectively scale to petabits per second”, Journal of Optical Networking, vol. 7, no. 10, pp. 876-894, October 2008

[5] E. Yetginer, and G. Rouskas, "Power Efficient Traffic Grooming in Optical WDM Networks”, in Proc. of IEEE GLOBECOM 2009, pp. 16, Nov.-Dec. 2009

[6] Slaviša Aleksic, “Analysis of Power Consumption in Future HighCapacity Network Nodes”, Journal of Optical Communications and Networking, vol. 1, no. 3, pp. 245-258, August 2009

[7] L. Chiaraviglio, M. Mellia, F. Neri, "Reducing Power Consumption in Backbone Networks”, in Proc. of IEEE ICC 2009, pp. 1-6, Jun. 2009.

[8] P. Batchelor, B, Daino, P. Heinzmann, D. R. Hjelme, R. Inkret, G. H. A. Jäger, M. Joindot, A. Kuchar, E. Le Coquil, P. Leuthold, G. de Marchis, F. Matera, B. Mikac, H. -P. Nolting, J. Späth, F. Tillerot, B. Van Caenegem, N. Wauters, C. Weinert, "Study on the implementation of optical transparent transport networks in the European environmentResults of the research project COST 239", Photonic Network Communications, vol. 2, no. 1, pp 15-32, 2000.

[9] http://www.ict-bone.eu/portal/landing_pages/index.html

[10] T. Politi, A. Tzanakaki, M. O'Mahony, K. Katrinis, P. Van Daele, M. Pickavet, D. Simeonidou, A. Stavdas, G. Franzl, J. Mitchell, P. Castoldi, S. Spadaro, F. Callegatti, P. Petropoulos, K. Vlachos, A. Pattavina, ICTON 2010, "ICT BONE views on the Network of the Future: the role of optical networking”, (Invited paper), ICTON 2010, Munich, Germany, pp. 1 - 4, June 2010

[11] T. Politi, M. J. O'Mahony, A. Tzanakaki, K. Katrinis, F. Callegatti, P. Castoldi, J. Mitchell, K. Vlachos, P. Petropoulos, M. Pickavet, D. Simeonidou, A. Pattavina, G. Maier, et al, 2nd report on dissemination activities including updated roadmap, BONE, http://www.ictbone.eu/portal/landing_pages/bone_deliverables/bone_216863_-_d014_-_report_y2.pdf

[12] B. Mukherjee, "Optical WDM Networks", Springer Verlag, ISBN-9780-387-29055-3, 2006

[13] J. Baliga, R. Ayre, K. Hinton, W. V. Sorin, R. S. Tucker, "Energy Consumption in Optical IP Networks", in IEEE/OSA Journal of Lightwave Technology, vol. 27, no. 13, pp. 2391-2403, 2009.

[14] K. Katrinis and A. Tzanakaki, "On the Dimensioning of WDM Optical Networks with Impairment-aware Regeneration", in IEEE/ACM Transactions on Networking, 2011 (to appear)

[15] Gurobi Optimizer 2.0, http://www.gurobi.com/ (online source)

[16] I. Morita, “Advanced Modulation Format for 100-Gbit/s Transmission”, TuE3.3 (Invited), LEOS Summer Topical Meeting, pp. 252 - 253, 2007

[17] H. Bulow, T. Rankl, "Soft coded modulation for sensitivity enhancement of coherent 100-Gbit/s transmission systems", OFC 2009, JThA46, pp. $1-3$

[18] S. Aleksic, "Power efficiency of $40 \mathrm{Gbit} / \mathrm{s}$ and $100 \mathrm{Gbit} / \mathrm{s}$ optical ethernet ”, ICTON 2009, pp. 1-1, 2009

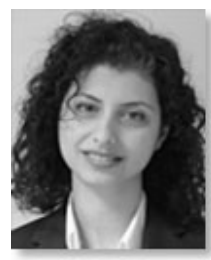

Anna Tzanakaki is an Associate Professor at the Athens Information Technology (AIT), where she is leading the Network Design and Services (NDS) research group. She is also an adjunct faculty member of the Information Networking Institute of Carnegie Mellon University, USA.

She has obtained a BSc degree in Physics from the University of Crete, Greece, an MSc and a PhD both from the University of Essex, UK. She is a co-author of over 100 publications in international journals and conferences. She is a co-inventor of 1 granted and 11 published patents. She is a technical referee for various journals and conferences. Her research interests include resource end energy efficient optical networks, cross-layer resilient network design and traffic provisioning and the Future Internet.

Dr. Tzanakaki is a senior member of the IEEE and has served as member of several Technical Program Committees.

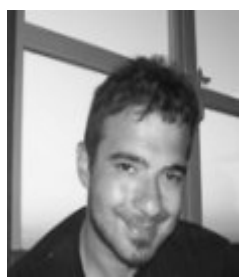

Kostas M. Katrinis is with the Network Design and Services (NDS) group at the Athens Information Technology (AIT), Athens, Greece. In the past, he has worked as a research assistant at the Communication Systems Group at ETH Zurich in the area of multimedia communication. He has authored or co-authored numerous publications in international journals and conferences and two book chapters. His research interests are in the area of network planning, sustainable networking, trustworthy communication and network performance evaluation.

He received the diploma in Computer Engineering from the Computer Engineering and Informatics Dept., University of Patras, Patras, Greece in 2000 and the PhD in Technical Sciences from the Swiss Federal Institute of Technology (ETH Zurich), Zurich, Switzerland in 2006.

Dr. Katrinis is a member of the Technical Chamber of Greece since 2000

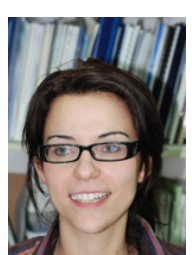

Christina (Tanya) Politi received a B.Sc. in physics from the University of Athens in 1998 and a M.Sc. degree in the "Physics of laser communications" from the University of Essex in 2000. Subsequently, she joined the Photonic Network Research Group in the Department of Electronic Systems Engineering at the University of Essex where she worked until early 2005 and obtained her $\mathrm{PhD}$. Today she is an Assistant Professor at the University of Peloponnese. She is a co-author of over 50 
international journal and conference publications in the area of optical communication systems.

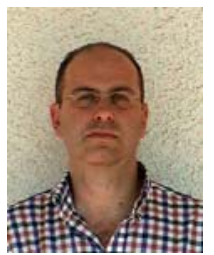

Alex Stavdas B.Sc. in Physics (University of Athens), M.Sc. in Optoelectronics and Laser Devices (Heriot-Watt University/St-Andrews University), Ph.D. (University College London) in the field of wavelength routed WDM networks. $\mathrm{He}$ is the author or co-author of over 80 journal publications and conference articles. $\mathrm{He}$ is Associate Professor in the Department of Telecommunications Science and Technology, University of Peloponnese, Greece. He served as Technical Program Committee Chairman and Member in a large number of International Conferences. Current interests include optical networking architectures, physical layer modeling of optical networks, ultrahigh capacity end-to-end optical networks, OXC architectures, Optical Packet/Burst Switching and WDM access networks.

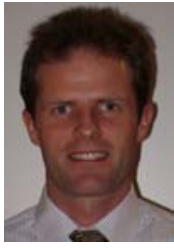

Mario Pickavet is full professor at Ghent University - IBBT. His current research interests are related to broadband communication networks (WDM, IP, (G-)MPLS, Ethernet, OPS, OBS) and include design, long-term planning, technoeconomical analysis and energy efficiency of core and access networks. Special attention goes to Operations Research techniques that can be applied for routing and network design. In this context, he is currently involved in several European and national projects, such as the Network of Excellence "Building the Future Optical Network inEurope" (BONE), DICONET and ECODE.

He has published about 200 international publications, both in journals (IEEE JSAC, IEEE Comm. Mag., Journal of Lightwave Technology, Eur. Trans. On Telecommunications, Photonic Network Communication, Journal of Heuristics,...) and in proceedings of conferences. He is co-author of the book 'Network Recovery: Protection and Restoration of Optical, SONET-SDH, IP, and MPLS'.

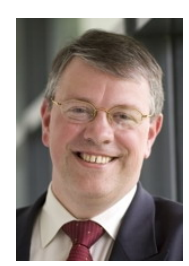

Peter Van Daele obtained a $\mathrm{PhD}$ in Electrical Engineering in 1988 at Ghent University. Since 1984 he works at the Department of Information Technology (INTEC) of Ghent University. From 1988 he is a permanent member of staff from IMEC at INTEC. Since April 1st 1993, he is part time professor at the University of Gent and is since 2004 also involved in the IBBT. He was and is directly responsible for several RACE, ACTS, ESPRIT, IST and ICT-projects such as the FP6-project BREAD on "Broadband for All" and the FP7 Network of Excellence "BONE".

Peter Van Daele is author or co-author of about 400 publications and was and is involved in the organization of several major international conferences such as ECOC (European Conference on Optical Communications), BBEurope (Broadband Europe) and SPIE Photonics Europe.

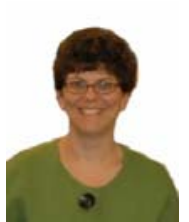

Dimitra Simeonidou is the Head of the High Performance Networks Group, which includes the Photonics Networks Laboratory and the newly established Network Media Laboratory, at the University of Essex, UK. She joined Essex in 1998 (previously with Alcatel Submarine Networks). While at Alcatel, she held the post of Senior Principle Engineer and contributed to the introduction of WDM in long-haul submarine links and pioneered the design and deployment of optical add-drop multiplexers. At Essex, she is leading a group of 30 researchers and Ph.D. students and she is involved in numerous national and international research projects.
Her research is focusing in the fields of optical networks, grid and cloud computing, and the future Internet. She is author and coauthor of over 350 papers, 11 patents, and several standardization documents.

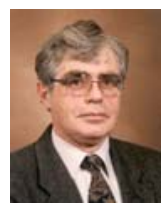

Mike O Mahony In the period 1980-1991, Mike O Mahony was Head of Section at British Telecom Research Labs, responsible for research into terrestrial long haul optical systems and networks. Areas of research included optical amplifiers, coherent optics, pico-second pulse systems and optical networks. In 1991 he joined the Department of Electronic Systems Engineering at the University of Essex as Professor of Optical Communication Networks. He was Head of Department from 1996-1999. Currently Emeritus Professor associated with the High Performance Networking Group within the School of Computer Science \& Electronic Engineering. Principle interests concern the study of the network infrastructures and optical technologies needed to support high performance networking.

Professor O' Mahony is the author of over 250 papers relating to optical communications.

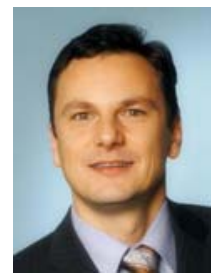

Slaviša Aleksić received his Dipl.-Ing. and Ph.D. degrees in electrical engineering from Vienna University of Technology, Austria, in 1999 and 2004, respectively. His current research interests include communication networks, photonic networks, energy efficiency in communication networks, high-speed optical and electrical signal processing systems, as well as high-speed media access control (MAC) protocol design and implementation. He is author or co-author of more than 70 scientific publications including book chapters, papers in peerreviewed scientific journals, and contributions to internationally recognized conferences. He has experience in both research and industrial fields through successfully managing and conducting many projects related to communication networks including two projects funded by the Austrian Science Fund (FWF) and a number of projects in collaboration with several Austrian and European academic institutions and companies. Dr. Aleksić is a member of the Institute of Electrical and Electronics Engineers (IEEE-USA), of the Austrian Electrotechnical Association (OVE-Austria), and of the Institute of Electronics, Information and Communication Engineers (IEICE-Japan). He has received several international awards, grants, and recognitions.

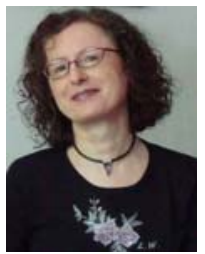

Lena Wosinska received her $\mathrm{Ph} . \mathrm{D}$. degree in photonics and Docent degree in optical networking from the Royal Institute of technology KTH. She joined KTH in 1986, where she is currently associate professor in the School of Information and Communication Technology (ICT), heading a research group in optical networking (Negonet), and coordinating a number of national and international scientific projects. Her research interests include optical network management, reliability and survivability of optical networks, photonics in switching, and fiber access networks. She has been involved in a number of professional activities including guest editorship of several special issues that appeared in OSA Journal of Optical Networking. In 2007-2009 she has been an Associate Editor of OSA Journal of Optical Networking, and since April 2009 she serves on the Editorial Board of IEEE/OSA Journal of Optical Communications and Networking.

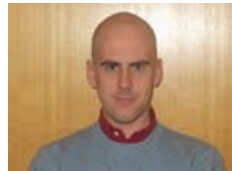

Paolo Monti received a Laurea degree in Electrical Engineering (2001) from the Politecnico di Torino, Italy, and a Ph.D. in Electrical Engineering (2005) from the 
University of Texas at Dallas (UTD). From 2006 to 2008 he worked as a Research Associate of the Open Networking Advance Research (OpNeAR) Lab at UTD. He joined the Royal Institute of Technology (KTH) in September 2008 where he is currently an Assistant Professor in the School of Information and Communication Technology (ICT/FMI) and a member of the Next Generation Optical Networks (NEGONET) group. He co-authored more than thirty papers published in international journals and presented in leading international conferences. His research interests include network planning, protocol design, performance evaluation and optimization techniques for both optical and wireless networks. 


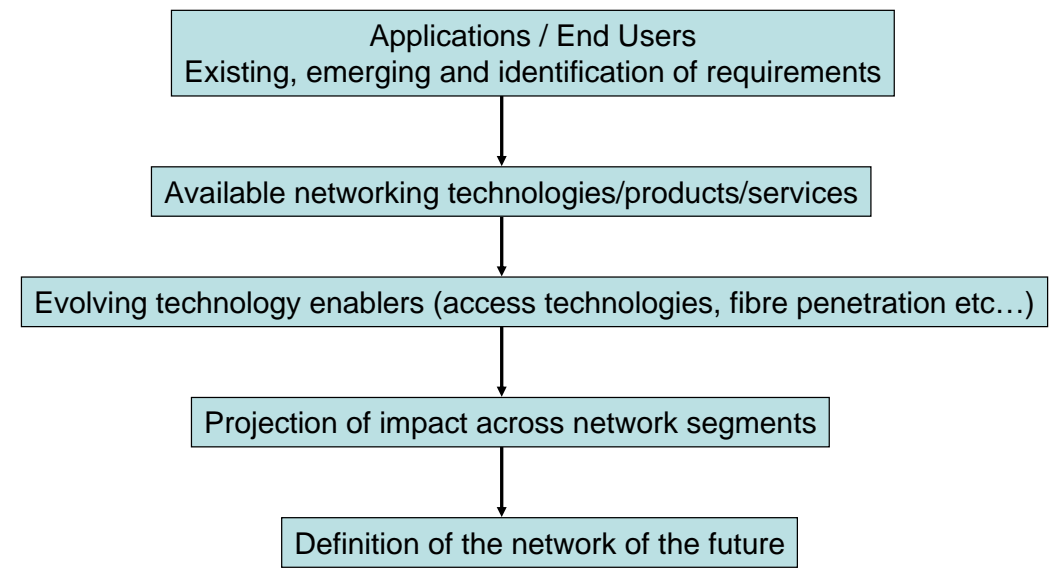

Figure 1. Roadmap methodology 


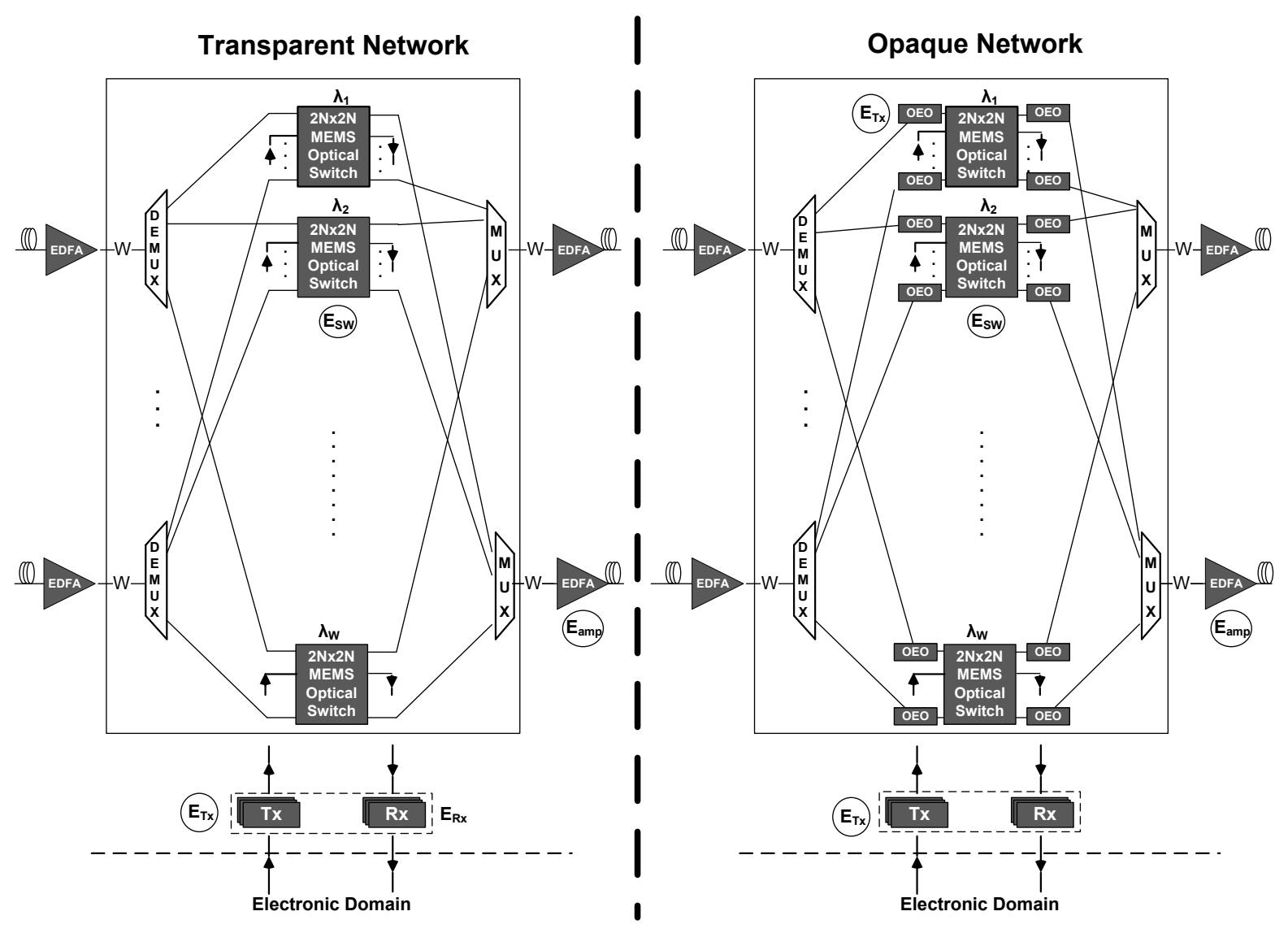

(a)

(b)

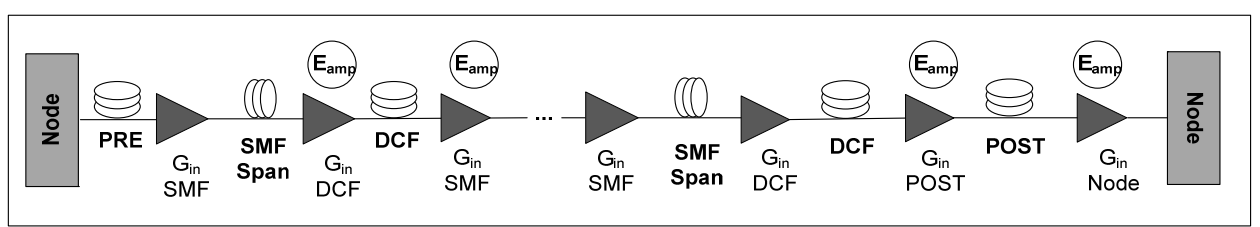

(c)

Figure 2. (a) Transparent and (b) Opaque OXC node and (c) transmission link model 

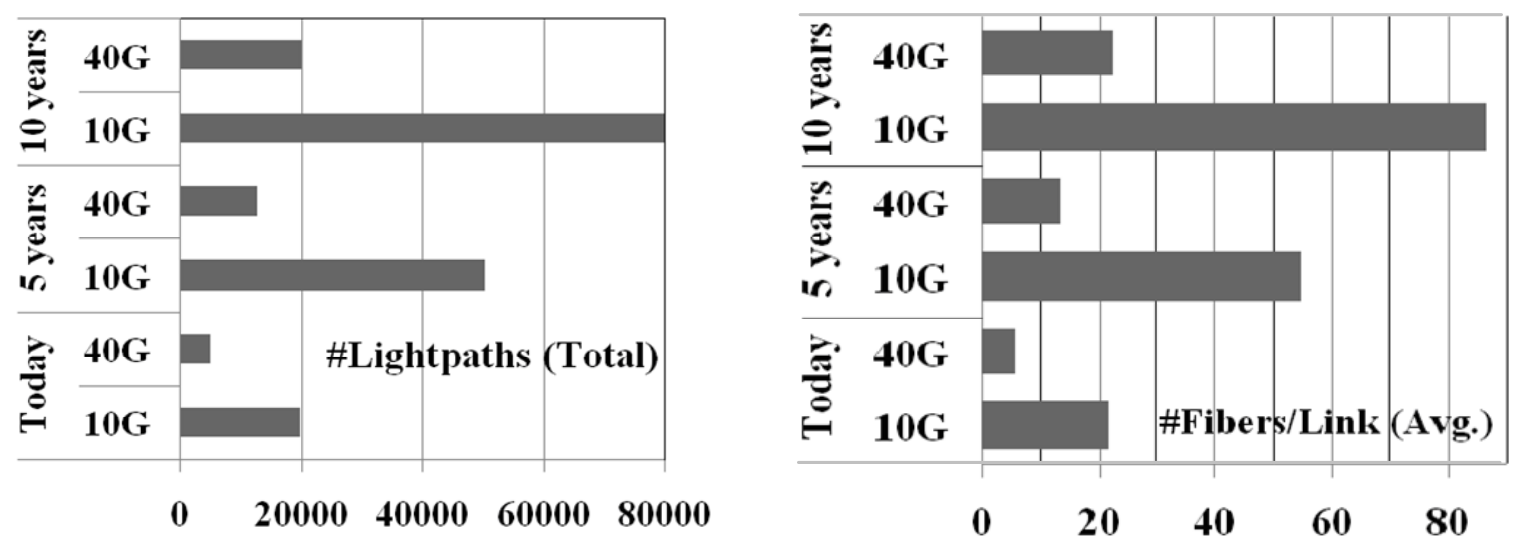

Figure 3. Metrics capturing the European optical backbone dimensions 


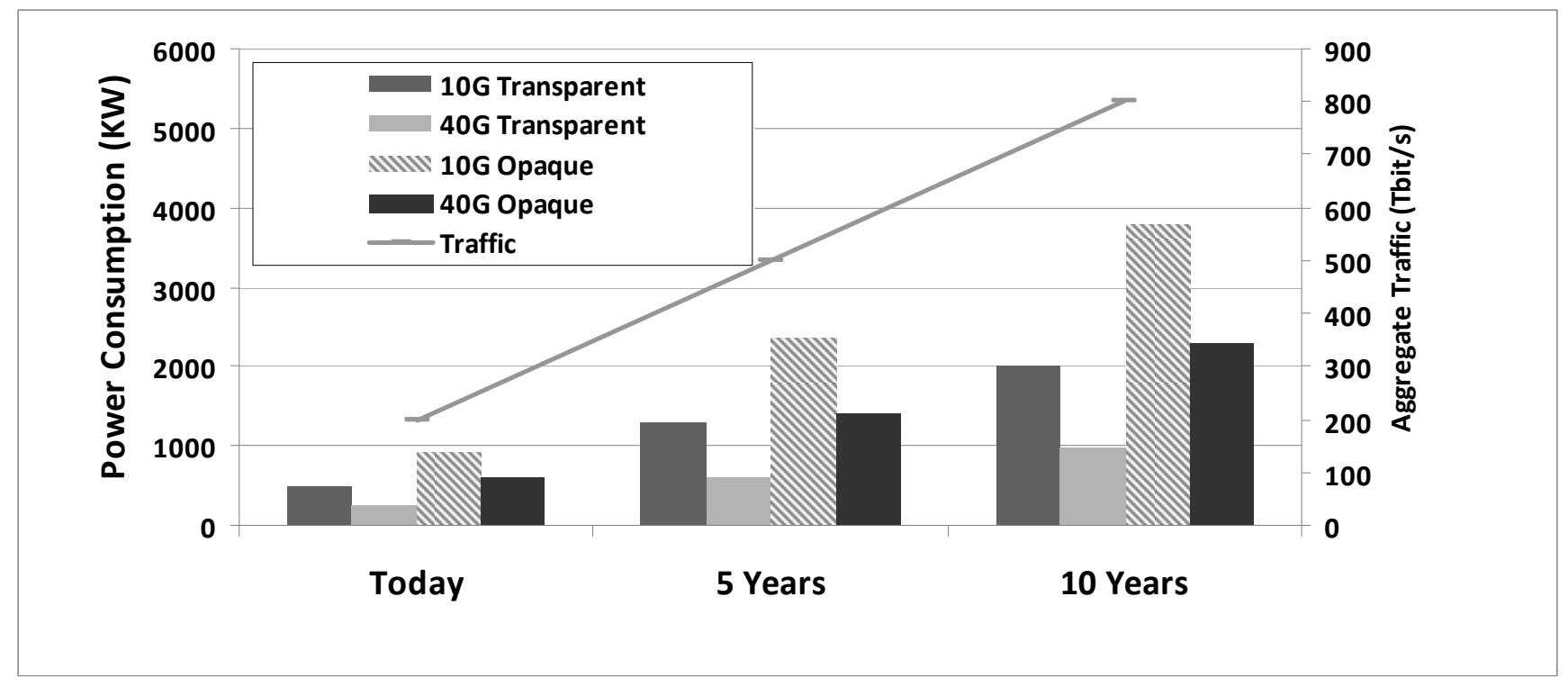

Figure 4. Total network power consumption for 10Gbit/s to $40 \mathrm{Gbit} / \mathrm{s}$ per wavelength, both for the transparent and opaque architectures together with the corresponding traffic volume carried by the network 


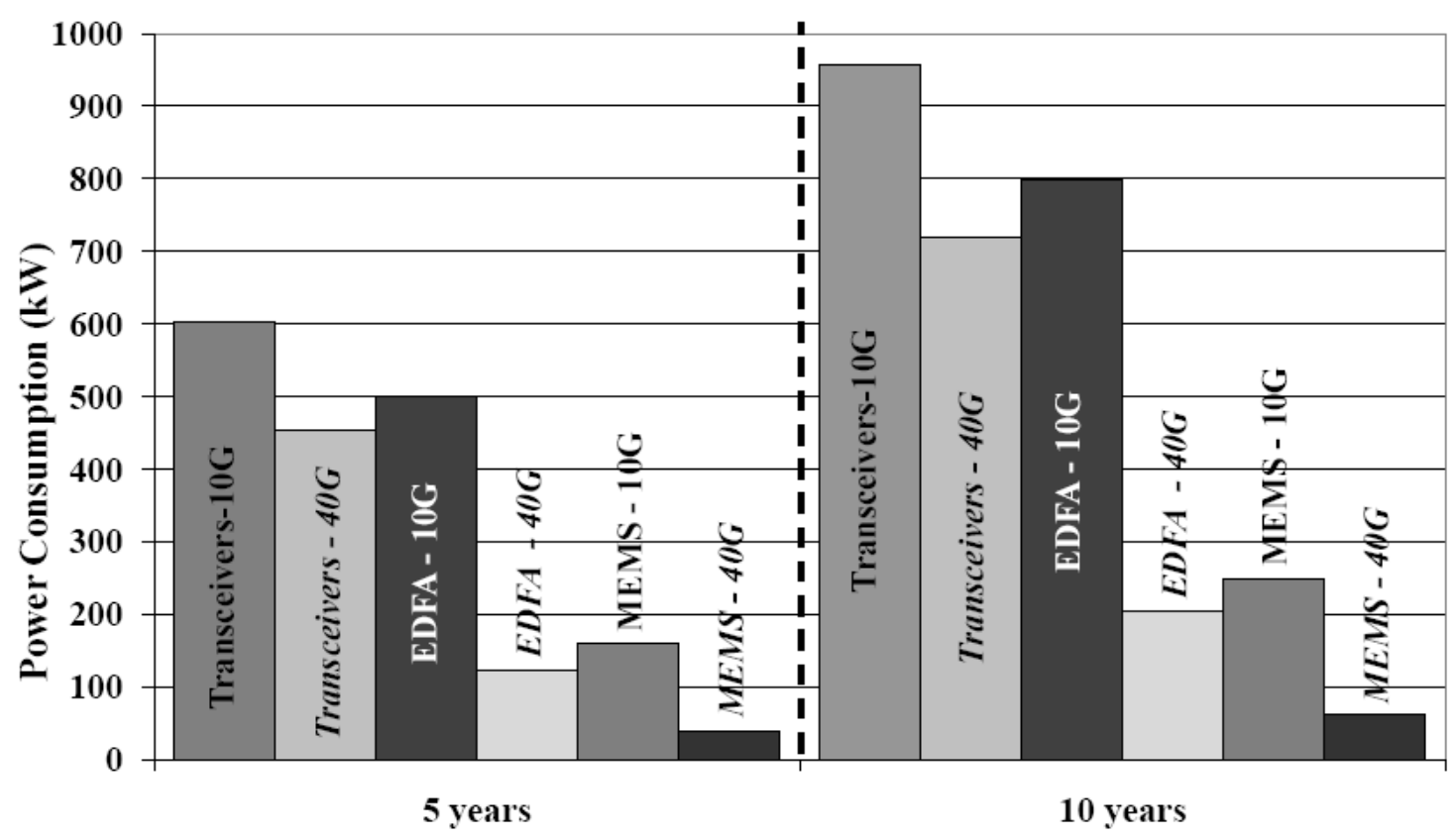

Figure 5. Power consumption of the various active network components for $10 \mathrm{Gbit} / \mathrm{s}$ and $40 \mathrm{Gbit} / \mathrm{s}$ per wavelength 
TABLE 1

TYPICAL POWER CONSUMPTION OF ACTIVE WDM NETWORK COMPONENTS

\begin{tabular}{|c|c|c|}
\hline \multirow{2}{*}{ Symbol } & Description & $\begin{array}{c}\text { Power } \\
\text { Consumption (W) }\end{array}$ \\
\hline \multirow{2}{*}{$E_{T x}$} & O/E/O: Line-Side Transceivers (WDM Long Reach - 2500km) \\
\cline { 2 - 3 } & O/E/O: Switch-Side Transceivers (Short Reach) \\
(10G) & 6 \\
\hline \multirow{2}{*}{$E_{T x}$} & O/E/O: Line-Side Transceivers (WDM Long Reach - 1800km) \\
\cline { 2 - 3 } & O/E/O: Switch-Side Transceivers (Short Reach) \\
(40G) & 1 \\
\hline$E_{a m p}$ & EDFA (20dBm) & 18 \\
\hline$E_{S W}^{N}(\mathrm{~N}=8)$ & 8x8 2D MEMS Switch & 13 \\
\hline$E_{S W}^{N}(\mathrm{~N}=16)$ & 16x16 2D MEMS Switch & 14 \\
\hline$E_{S W}^{N}(\mathrm{~N}=32)$ & 32x32 3D MEMS Switch & 14 \\
\hline$E_{S W}^{N}(\mathrm{~N}=64)$ & 64x64 3D MEMS Switch & 25 \\
\hline
\end{tabular}

